\title{
Stabilizing Magamp Control Loop by Using an Inner-Loop Compensation
}

\author{
Chao-Hwa Yang, Dan Y. Chen, Senior Member, IEEE, Cliff Jamerson, and Yan Pei Wu
}

\begin{abstract}
By using the inner-loop compensation scheme proposed in this paper, the resonance peaking of the open-loop transfer function of a magamp circuit is eliminated. This makes the loop compensation of magamp control much easier. If properly used, the proposed scheme is capable of making the resultant loop gain essentially independent of parameter variation of the saturable reactors.
\end{abstract}

\section{INTRODUCTION}

A MAGAMP post regulator is often the preferred choice for many high performance multi-output power supply applications. The major attraction of magamp is that the voltages of a high-current multi-output supply can be independently regulated with efficiency and reliability. Recent papers on this subject reflect the surge of interest in this area [1]-[10].

One of the major tasks in the design of magamp regulators is to compensate the control loop to achieve loop stability and regulation performance. There have been several papers deling with this subject published recently [1]-[3]. In the conventional compensation scheme, the open-loop transfer function is a second order function normally with resonance peaking. To make things worse, the resonance frequency, in some magamps, is sensitive to the parameter variation of the saturable reactor and lineload condition [2]. Compensation of the magamp control circuit, therefore, become rather annoying. The resultant loop performance is sensitive to circuit parameter changes. The major parameter variations are the ESR of the output filter capacitor, the inductance of the choke and the permeability of the saturable reactor.

To rectify this situation, a new compensation scheme is proposed in this paper. By using this new scheme, the open loop transfer function is reduced from second order to first order, therefore the resonance peaking phenomenon is eliminated. If properly designed, the proposed scheme is capable of making the resultant loop gain essentially independent of the power circuit parameters.

In the paper, a review of the problems associated with a conventional compensation scheme is given first. The

Manuscript received March 8. 1990; revised August 28. 1990

C.-H. Yang and Y. P. Wu are with National Taiwan University, Taipei, Taiwan.

D. Y. Chen is with the Bradley Department of Electrical Engineering. Virginia Polytechnic Institute and State University. Blacksburg. VA 24061.

C. Jamerson is with NCR Power Systems, Lake Mary. FL.

IEEE Log Number 9100536 new compensation scheme is then proposed, analyzed and implemented in a circuit. Experimental verification is given and a practical design procedure is illustrated in an example.

\section{Review of Control Loop Behavior of a MAGAMP USING A CONVENTIONAL} COMPENSATION SCHEME

Fig. I shows a circuit diagram of a PWM-controlled forward converter with a magamp post regulator. Fig. 2(a) shows the circuit diagram of a conventional magamp feedback control circuit with self reset from the regulated output. This paper is focused on this common-used reset scheme. Fig. 2(b) shows the control block diagram of the same circuit. The functional dependence of the small signal transfer functions $F_{R}, F_{M}$ and $F_{F}$ are expressed in (1)(4):

$$
\begin{aligned}
F_{R} & =\frac{\hat{I}_{R}}{\hat{V}_{E}}\left(\begin{array}{c}
\text { control voltage-to-reset current } \\
\text { transfer function }
\end{array}\right) \\
& =\frac{-R_{B}}{\left(R_{B}+R_{S}\right) R_{E}} \\
F_{M} & \doteq \frac{\hat{d}}{\hat{I}_{R}}\left(\begin{array}{c}
\text { reset current-to-duty cycle } \\
\text { transfer function }
\end{array}\right) \\
& =\frac{-0.4 \Pi \mu_{M} N^{2} A_{\ell} f_{s}}{l_{e} V_{x} \cdot 10^{8}}
\end{aligned}
$$

where

$$
\mu_{M}=\frac{(\Delta B)^{2} f_{s}}{K_{c^{\prime}} \cdot P_{L} \cdot 10^{6}}
$$

where

$$
\begin{aligned}
K_{c} & =1.2 \text { for Square } 80 \text { permalloy } \\
K_{c} & =1.08 \text { for Magnetic Inc. } 2704 \text { metglas } \\
F_{F} & =\frac{\hat{V}_{o}}{\hat{d}} \text { (duty cycle-to-output transfer function) } \\
& =V_{x}\left[\frac{1+S R_{C} C}{1+S\left(R_{C} C+L / R_{L}\right)+S^{2} L C}\right]
\end{aligned}
$$

for continuous mode. 


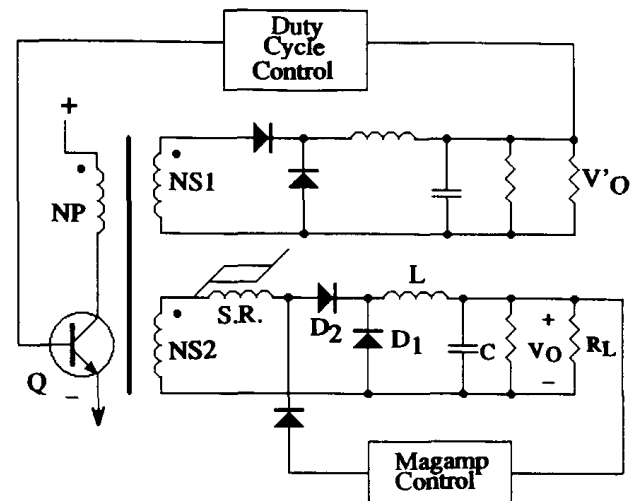

Fig. 1. PMW-controlled forward converter with magamp post regulator.

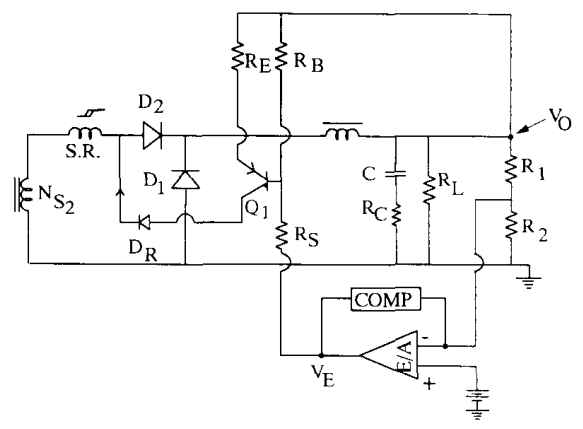

(a)

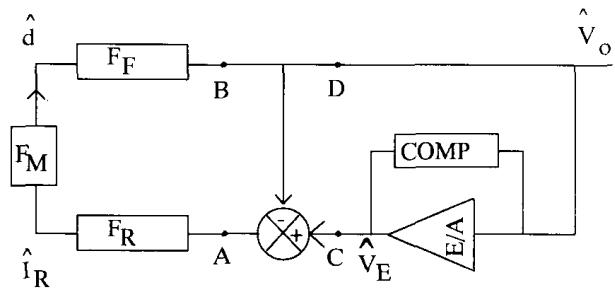

(b)

Fig. 2. (a) Magamp control circuit diagram. (b) Control block diagram.

It is to be noted that $F_{R}$ is a function of the reset circuit resistor values; $F_{M}$ is a function of saturable reactor parameters, dc operating condition and switching frequency; and $F_{F}$ is a function of power circuit parameters and d.c. operating condition. For detailed derivation of these transfer functions, readers should refer to [1].

Referring to Fig. 2(b), there are two closed loops in the diagram. The inner-loop consists of $F_{R}, F_{M}, F_{F}$ and the feedback, and the outer loop consists of $F_{R}, F_{M}, F_{F}$ and the error amplifier $E / A$. The Bode plots of the two openloop transfer functions are given in Fig. 3, where $F_{R} F_{M} F_{F}$ is the open-loop transfer function of the inner-loop (between points $A$ and $B)$ and $G\left(=F_{R} F_{M} F_{F} / 1+F_{R} F_{M} F_{F}\right)$ is the open-loop transfer function of the outer loop (between points $C$ and $D$ with the inner-loop closed). $G(s)$ is

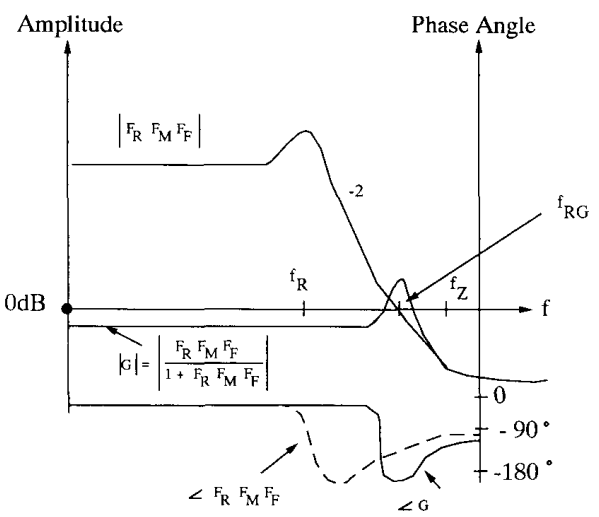

Fig. 3. Bode plot of open loop transfer function of conventional scheme.

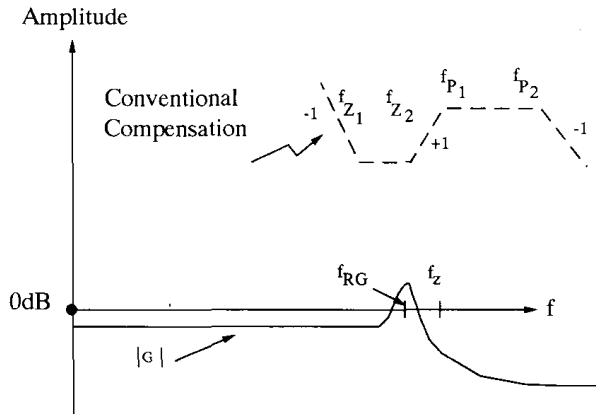

Fig. 4. Placement of poles and zeros in conventional compensation scheme.

a second order transfer function with low frequency gain less than $0 \mathrm{~dB}$ and the resonance frequency $f_{R G}$ is given by (5), [2]:

$$
f_{R G} \cong \frac{1}{2 \Pi \sqrt{L C}}\left[1+F_{R} F_{M} F_{F}(0)\right]^{1 / 2} .
$$

It is to be noted that the resonance peaking of $G$ occurs near the $0 \mathrm{~dB}$ crossover frequency of $F_{R} F_{M} F_{F}$ and the peaking occurs because $F_{R} F_{M} F_{F}$ crossovers $0 \mathrm{~dB}$ with -2 slop [2].

\section{Conventional Compensation Scheme}

The placement of zeros and poles of a conventional compensation scheme is shown by the dotted line in Fig. 4. The two zeros, $f_{Z 1}$ and $f_{Z 2}$, are placed near the resonance peaking frequency $f_{R G}$ and the pole $f_{p 1}$ is placed near the capacitor ESR zero $f_{Z}$ of the power circuit. The second pole $f_{p 2}$ is used to suppress switching frequency noise. There are practical difficulties of compensating a magamp circuit this way because of the nature of the two complex poles associated with resonance frequency $f_{R G}$. First, $f_{R G}$ is highly dependent on saturable reactor parameters and line-load conditions. This is apparent from (5) and the 
discussion of functional dependence of transfer functions given earlier in this section. Second, the $Q$ factor of the transfer function $G(s)$, in some cases, is even higher than the $Q$ factor of the original transfer function $F_{R} F_{M} F_{F}$ determined by the power circuit parameters. This makes the peaking of $G(s)$ more pronounced and the changing of the phase angle more rapid near resonance peaking frequency. Both difficulties described above requires conservative placement of compensating zeros to stabilize the system. In doing so, loop gain performance is sacrificed.

\section{Proposed Compensation Scheme}

To improve the control performance, a new compensation scheme is proposed as follows. Compensation is added to both the $F_{R}$ block and the $E / A$ block in Fig. 2(b). The former is referred to as inner-loop compensation and the latter as outer-loop compensation. Both are discussed in the following.

\section{Inner-Loop Compensation}

The transfer function of the desired inner-loop compensation $F_{R}^{\prime}$ is shown by the dotted line in Fig. 5(a). A compensation zero $F_{Z 1}$ is placed near resonant frequency $f_{R}$ of $F_{F}$ and a compensation pole $f_{p 1}$ is placed near capacitor ESR zero of $F_{F}$. It is to be noted that the resonance frequency $f_{R}$ is a function of $L$ and $C$ and is independent of saturable reactor parameters. Therefore, the placement of $f_{p 1}$ is relatively straight forward. With the compensation, the magnitude of open inner-loop transfer function $\left|F_{R}, F_{M} F_{F}\right|$ is shown by the dotted line in Fig. 5(b). Notice that $\left|F_{R^{\prime}}, F_{M} F_{F}\right|$ has a -1 slop at zero $\mathrm{dB}$ crossover. This is true if the low frequency gain $\left|F_{R^{\prime}}(0) F_{M} F_{F}(0)\right|$ is much greater than one, which normally is the case.

The open outer-loop transfer function $\left|G^{\prime}\right|$ is shown by the solid line in Fig. 5(b). Notice that $G^{\prime}$ has essentially become a single-pole transfer function. The pole frequency $f_{P G^{\prime}}$ can be expressed as follows:

$$
f_{P G^{\prime}} \cong \frac{1}{2 \pi \sqrt{L C}} \cdot \frac{F_{R} F_{M} F_{F}(0)}{2} \cdot\left[1+\sqrt{\frac{4}{F_{R} F_{M} F_{F}(0)}}\right] .
$$

It is to be noted that the compensation zero (a real zero) $f_{Z I}$ cannot completely cancel out a resonance pole (complex pole) at $f_{R}$. Therefore, $F_{R^{\prime}} F_{M} F_{F}$ is not exactly a first order transfer function and $G^{\prime}$ is not either. For theoretical discussion of $G^{\prime}$ functional behavior and the derivation of (6), one should refer to the Appendix. However, it is concluded from the Appendix that $G^{\prime}$ is essentially a first-order transfer function and the outer-loop compensation will be done accordingly.

\section{Outer Loop Compensation}

Because $G^{\prime}(s)$ is essentially a first order transfer function, the outer-loop compensation is relatively straight forward. Fig. 6 shows two examples of the placement of compensation transfer functions.

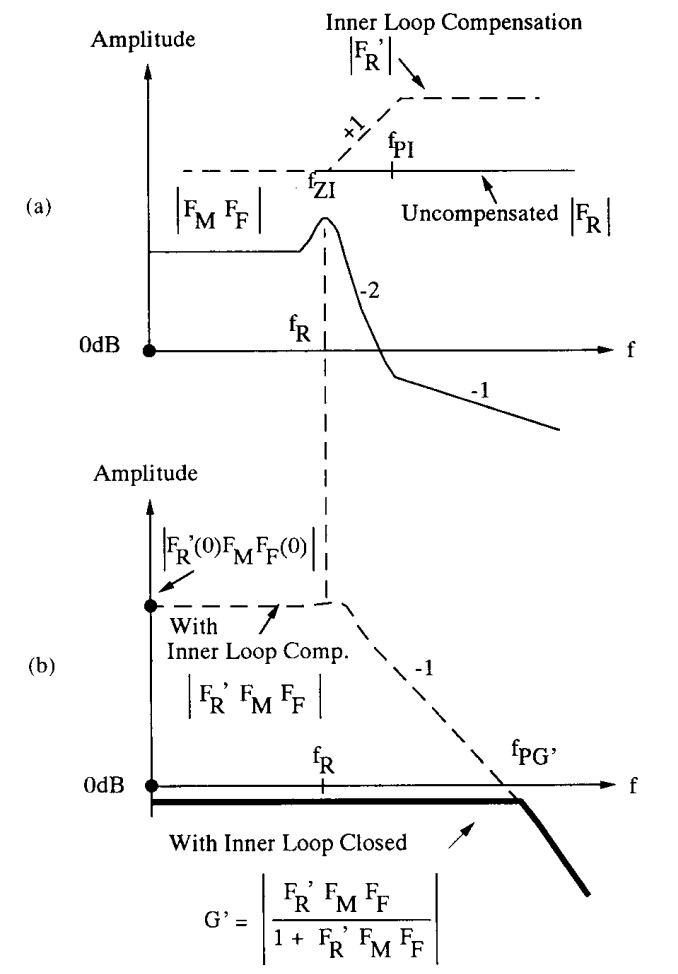

Fig. 5. (a) Placement of pole and zero in proposed inner loop compensation. (b) Magnitude plot of open-loop transfer function with inner loop compensation.
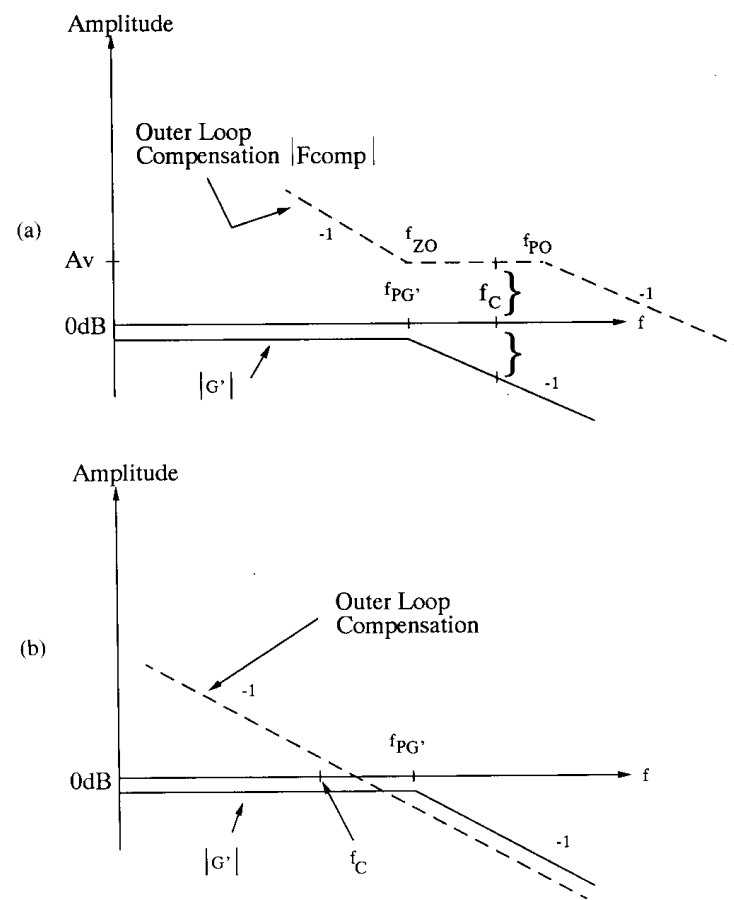

Fig. 6. Placement of poles and zeros in proposed outer loop compensation. 
Lead-Lag Compensation: In the case of Fig. 6(a), the desired loop gain crossover frequency $f_{C}$ is greater than $f_{P G^{\prime}}$. A compensation zero $f_{Z O}$ is placed near $f_{P G^{\prime}}$ and a compensation pole $f_{P O}$ is placed for attenuating high frequency noise. From (6), it can be seen that $f_{P G^{\prime}}$ is a function of saturable reactor parameters and the line-load conditions. Therefore, the placement of $f_{Z O}$ must take the worst condition into consideration similar to the scheme used in a conventional compensation. However, $G^{\prime}$ in this case is a single-pole transfer function, unlike the $G$ in a conventional scheme in which there is a resonance peaking. Therefore, the placement of $f_{z o}$ is less critical. Furthermore, because of single-zero compensation as opposed to two-zero compensation as required in a conventional scheme, the low-frequency loop gain of the new scheme usually ends up higher.

Dominant Pole Compensation: In the case of Fig. 6(b), where desired crossover frequency $f_{C}$ is less than $f_{P G^{\prime}}$, a dominant pole compensation is used. The main advantage of dominant pole compensation is that the overall loop gain is made essentially independent of saturable reactor parameters and line-load conditions. This is a significant advantage in a high volume production situation. The crossover frequency in this case is lower than that of leadlag compensation. However, it can seen from (6), if the inner open loop gain $F_{R}^{\prime}(0) F_{M} F_{F}(0)$ is designed large, $f_{P G}$, is large to begin with and the sacrifice in crossover frequency is minimal.

\section{Circuit Implementation}

Fig. 7 shows the circuit implementation of the proposed scheme. The inner loop compensation is achieved by $C_{E}$ and $R_{3}$. The addition of $C_{E}$ and $R_{3}$ effectively changes the reset circuit transfer function from $F_{R}$ to $F_{R}^{\prime}$ discussed earlier. The outer-loop compensation is achieved by either a lead-lag compensation or a dominant pole compensation in the error amplifier. The determination of the compensation component values is discussed below.

Determination of $C_{E}$ and $R_{3}$ : With the inner-loop compensation, the transfer function $F_{R}^{\prime}$ is shown in (7) from which the zero $\left(f_{Z I}\right)$ and the pole $\left(f_{P I}\right)$ frequency can be found. From the discussion in the last two sections, the inner-loop compensation zero $f_{Z I}$ should be placed at the resonant frequency $f_{R}$ as indicated by (8). The pole frequency $f_{P I}$ should be placed at capacitor ESR zero frequency $f_{Z}$ as indicated by (9). Both (8) and (9) will be used later in the design example to determine $C_{E}$ and $R_{3}$ :

$$
\begin{gathered}
F_{R}^{\prime}=\frac{-R_{B}\left[\left(R_{E}+R_{3}\right) C_{E} S+1\right]}{\left(R_{B}+R_{S}\right) R_{E}\left(R_{3} C_{E} S+1\right)} \\
\frac{1}{2 \pi\left(R_{E}+R_{3}\right) C_{E}}=f_{Z I}=f_{R}=\frac{1}{2 \pi \sqrt{L C}} \\
\frac{1}{2 \pi R_{3} C_{E}}=f_{P I}=f_{Z}=\frac{1}{2 \pi R_{C} C} .
\end{gathered}
$$
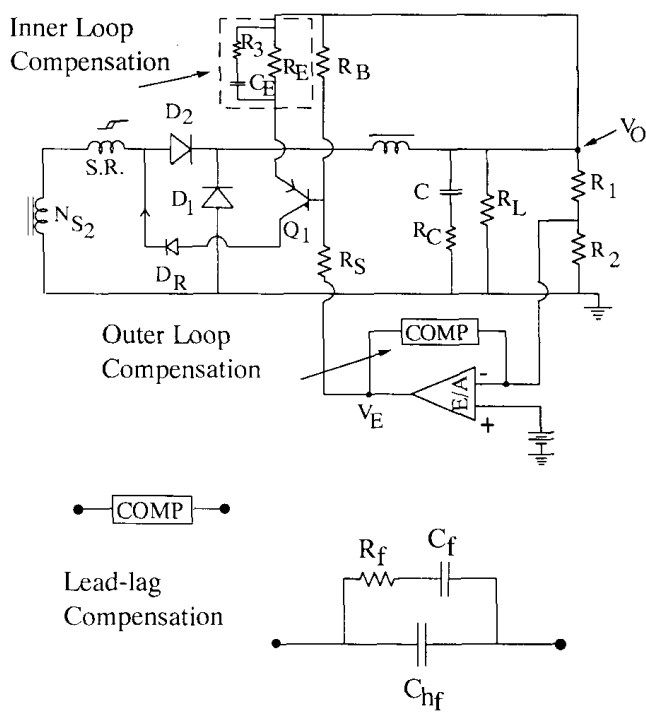

Dominant-pole Compensation

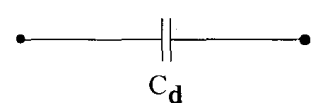

Fig. 7. Circuit implementation of proposed compensation scheme.

Determination of $R_{f}, C_{f}$ and $C_{h f}$ (Lead-Lag Compensation) or $C_{d}$ (Dominant-Pole Compensation): For the leadlag compensation, the outer-loop compensation zero $f_{Z O}$ should be placed at $f_{P G^{\prime}}$ as indicated by (10) and the compensation pole $f_{P O}$ is placed to attenuate the high frequency noise. Equation (11) shows the functional dependence of $f_{P O}$ on the compensation network parameters. Equation (12) shows the mid-band gain $A v$ of the outerloop compensation network. These equations will be used later in the design example to determine $R_{f}, C_{f}, C_{h f}$ and $R_{i}$ :

$$
\begin{aligned}
\frac{1}{2 \pi R_{f} C_{f}} & =f_{Z O}=f_{P G^{\prime}} \\
\frac{1}{2 \pi R_{f} C_{f} C_{h f} /\left(C_{f}+C_{h f}\right)} & =f_{P O} \\
\frac{R_{f}}{R_{1}} & =A v .
\end{aligned}
$$

For the case of dominant-pole compensation, $C_{d}$ can be determined by the following equation:

$$
C_{d}=\frac{1}{2 \pi f_{C} R_{1}} .
$$

\section{Design Example}

Magamp Power Circuit and Circuit Parameters:

$$
\begin{aligned}
f s & =50 \mathrm{Khz} \\
L & =190 \mathrm{uh} \\
C & =220 \mathrm{uf}
\end{aligned}
$$




$$
\begin{aligned}
& R_{C}=0.1 \Omega \\
& R_{S}=1 \mathrm{k} \Omega \\
& R_{E}=47 \Omega \\
& V_{x}=72 \mathrm{~V} \\
& V_{O}=12 \mathrm{~V} \\
& R_{L}=3 \Omega .
\end{aligned}
$$

Saturable Reactor Parameters

$$
\begin{aligned}
N & =38 \\
A_{e} & =0.076 \mathrm{~cm}^{2} \\
l e & =6.18 \mathrm{~cm} .
\end{aligned}
$$

Material: Square 80 Permalloy (Mag. Inc. 50B10-1D). Desired loop-gain crossover frequency $f_{c}=56 \mathrm{kHz}$ Find the compensation parameters $R_{3}, C_{E}, R_{f}, C_{f}$ and $C_{h f}$. Step I: Calculate $F_{R} F_{M} F_{F}(0)$.

(a) From (1),

$$
\left|F_{R}\right|=\frac{1 \times 10^{3}}{(1+1) \times 10^{3} \cdot 47}=\frac{1}{94}
$$

(b) From (2)

$$
\left|F_{M}\right|=\frac{0.4 \pi \mu_{M} \cdot 38^{2} \cdot 0.076 \cdot 50 \times 10^{3}}{6.18 \cdot 72 \times 10^{8}}
$$

To find out $\mu_{M}, \Delta B$ must be found out first. Using Farady's Law,

$$
\begin{aligned}
\Delta B & =\frac{V_{x} \times 10^{8}}{N A_{e} f_{s}}\left(D_{Q}-\frac{V_{O}+V_{D}}{V_{x}}\right. \\
& =\frac{72 \times 10^{8}}{38 \cdot 0.076 \cdot 50 \times 10^{3}}\left(0.25-\frac{12+1}{72}\right) \\
& =3463 \mathrm{G} .
\end{aligned}
$$

Using $\Delta B / 2=1734 \mathrm{G}$ and $50 \mathrm{kHz}$ switching frequency, find core loss density $P_{L}=17 \mathrm{~W} / \mathrm{lb}$ from core catalog.

Using $\Delta B$ and $P_{L}$ values calculated above, determine $\mu_{M}$ from (3):

$$
\mu_{M}=\frac{(3462)^{2} \cdot 50 \times 10^{3}}{1.2 \cdot(17) \times 10^{6}}=29393 .
$$

Therefore,

$$
\begin{aligned}
F_{M} & =\frac{0.4 \pi \cdot(29393) \cdot 38^{2} \cdot 0.076 \cdot 50 \times 10^{3}}{6.18 \cdot 72 \times 10^{8}} \\
& =4.6 .
\end{aligned}
$$

(c) From (4),

$$
\begin{aligned}
F_{F}(0) & =V_{x}=72 \\
\therefore\left|F_{R} F_{M} F_{F}(0)\right| & =1 / 94 \cdot 4.6 \cdot 72=3.55 .
\end{aligned}
$$

Step II: Determine the inner-loop compensation parameters $R_{3}$ and $C_{E}$. (a) Placement of compensation zero $f_{Z I}$, using (8),

$$
\frac{1}{2 \pi\left(R_{E}+R_{3}\right) C_{E}}=\frac{1}{2 \pi \sqrt{L C}}=333 \mathrm{~Hz} .
$$

(b) Placement of compensation pole $f_{P I}$, using (9),

$$
\frac{1}{2 \pi R_{3} C_{E}}=\frac{1}{2 \pi R_{C} C}=1320 \text {. }
$$

(c) Solve for $C_{E}$ and $R_{3}$ from (13) and (14), noted that $R_{E}=47 \Omega$ from given specification:

$$
C_{E}=7.6 \mu \mathrm{f} \text { and } R_{3}=15.8 \Omega \text {. }
$$

Step III: Determine outer loop compensation parameters.

(a) Calculate $\left|G^{\prime}(0)\right|$ and $f_{P G^{\prime}}$. From (6),

$$
\begin{aligned}
\left|G^{\prime}(0)\right| & =F_{R^{\prime}}(0) F_{M} F_{F}(0) / 1+F_{R^{\prime}}(0) F_{M} F_{F}(0) \\
& =3.55 / 1+3.55=0.78=-2 \mathrm{db}
\end{aligned}
$$

From (6),

$$
f_{P G^{\prime}}=333 \cdot \frac{3.55}{2}\left[1+\sqrt{\frac{4}{3.55}}\right]=1434 .
$$

(b) Placement of compensation zero $f_{z o}$. Using (10),

$$
\frac{1}{2 \pi R_{f} C_{f}}=1434
$$

(c) Placement of compensation pole $f_{P O} \cdot f_{P O}$ is placed to attenuate the switching-frequency noise. It should be equal to or greater than the desired loop-gain crossover $f_{C}$ but should be significantly smaller than switching frequency. In this example, $5 \mathrm{kHz}$ is selected. Using (11),

$$
\frac{1}{2 \pi R_{f}\left(\frac{C_{f} \cdot C_{h f}}{C_{f}+C_{h f}}\right)}=5000 .
$$

(d) Sketch the approximated $G^{\prime}(s)$ using the results of Step III-(a).

(e) Sketch the desired transfer function Fcomp. Using the corner frequencies obtained from Step III(b) and (c) and set $|F \operatorname{comp}(f=5 \mathrm{kHz})|=\mid G^{\prime}(f=$ $5 \mathrm{kHz}$ ) in order to achieve the desired loop-gain crossover-hyphen frequency of $5 \mathrm{kHz}$. The result is similar to Fig. 6(a).

(f) From the Fcomp obtained in the last step, obtain mid-frequency gain $A v=14 \mathrm{~dB}$. Using (12), and set $A v=14 \mathrm{~dB}$,

$$
\frac{R_{f}}{R_{1}}=14 \mathrm{~dB} .
$$

(g) Determine $R_{1}$ and $R_{2}$. Assume the reference voltage of the operational amplifier is $2.5 \mathrm{~V}$.

From the consideration of the noise immunity and power dissipation in the two resistors $R_{1}$ and $R_{2}, R_{2}$ is selected to be $2.5 \mathrm{~K} \Omega . R_{1}$ can then be determined, by voltage divider equation, to be $9.5 \mathrm{~K} \Omega$. 


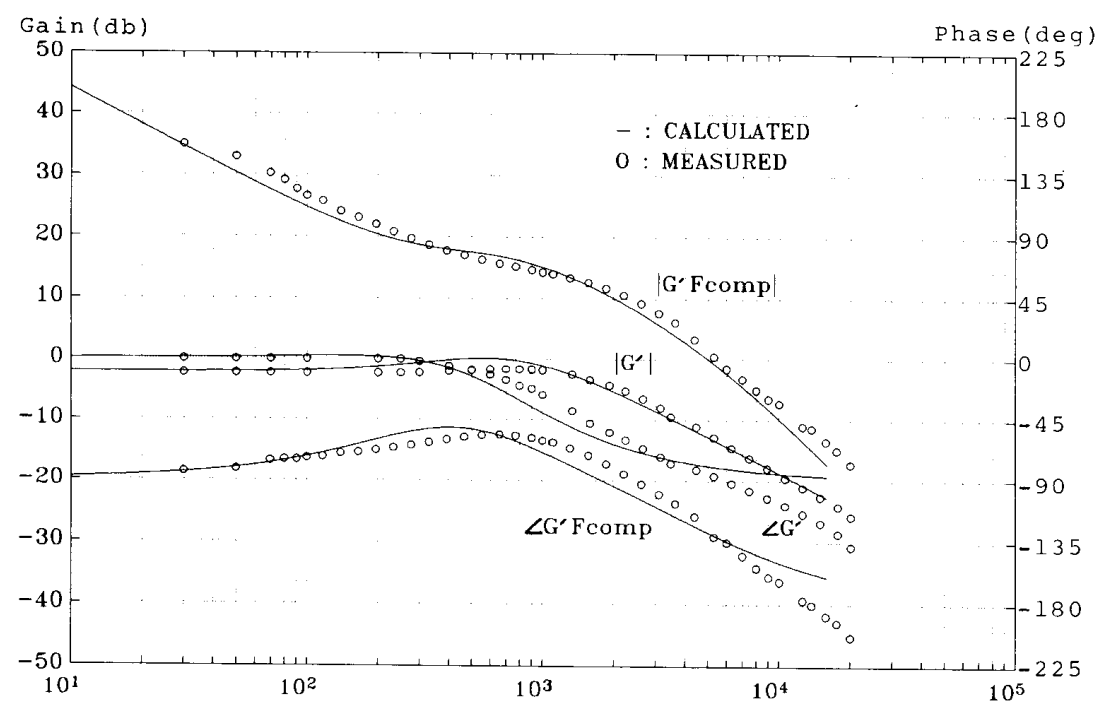

(a)

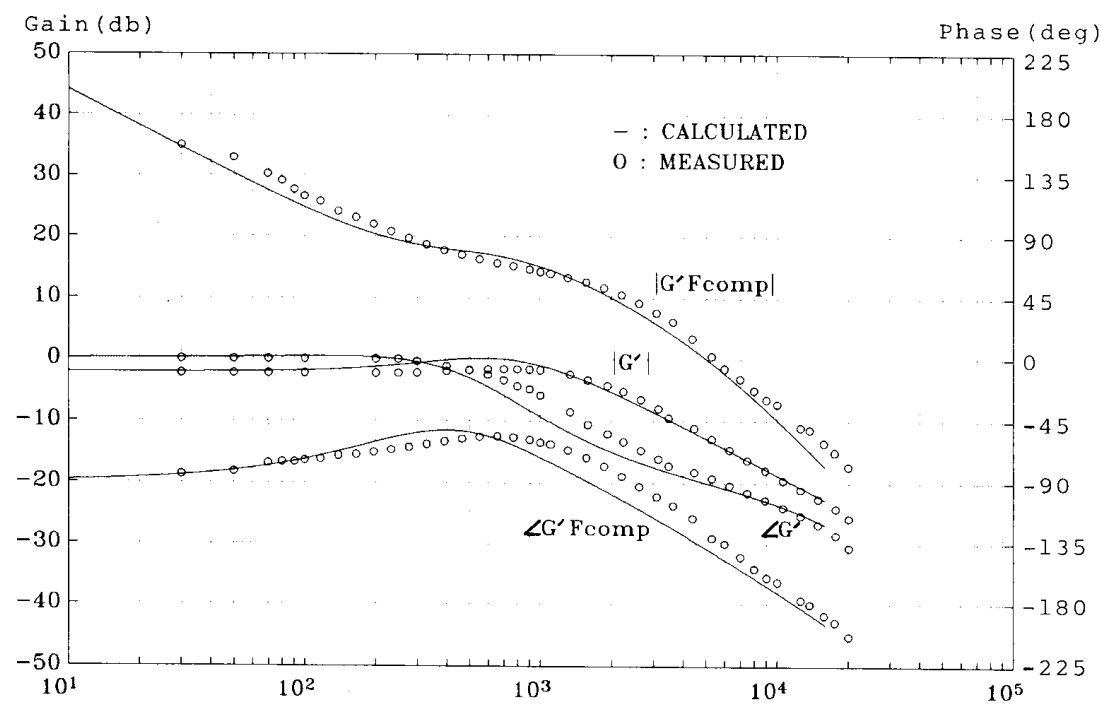

(b)

Fig. 8. Comparison of the predicted and measured $G^{\prime}$ function and $G^{\prime} F_{\text {comp }}$ function. (a) Neglecting phase delay in $F_{m}$. (b) Incorporating phase delay in $F_{M}$.

(h) Solve for $R_{f}, C_{h f}$. From (17),

$$
R_{f}=64 \mathrm{~K} \Omega
$$

From (15) and (16),

$$
C_{f}=7.5 \mathrm{nF} \text { and } C_{h f}=5.37 \mathrm{pF} .
$$

\section{Experimental Verification and Discussion}

Fig. 8(a) shows the comparion between the predicted and the measured $G^{\prime}$ function and the loop gain $G^{\prime} F$ comp. There is a good agreement between the two. Generally speaking, when the frequency exceeds $3 \mathrm{kHz}$, however, phase angle deviation becomes pronounced. This is due to the fact that the phase delay associated with the transfer function $F_{M}$ is neglected in this paper. The phase delay $\phi_{D}$ associated with $F_{M}$ can be approximated by the following relationship [9].

$$
\phi_{D}(\text { in radian })=-\left(2 D^{\prime}+\alpha\right) \frac{f}{f_{s}}
$$

where $D^{\prime}$ is the off duty cycle of the diode $D_{1}$ and $\alpha$ is factor associated with reactor resetting source. It ranges between 0 and 1. $\alpha=0$ for a perfect current resetting 


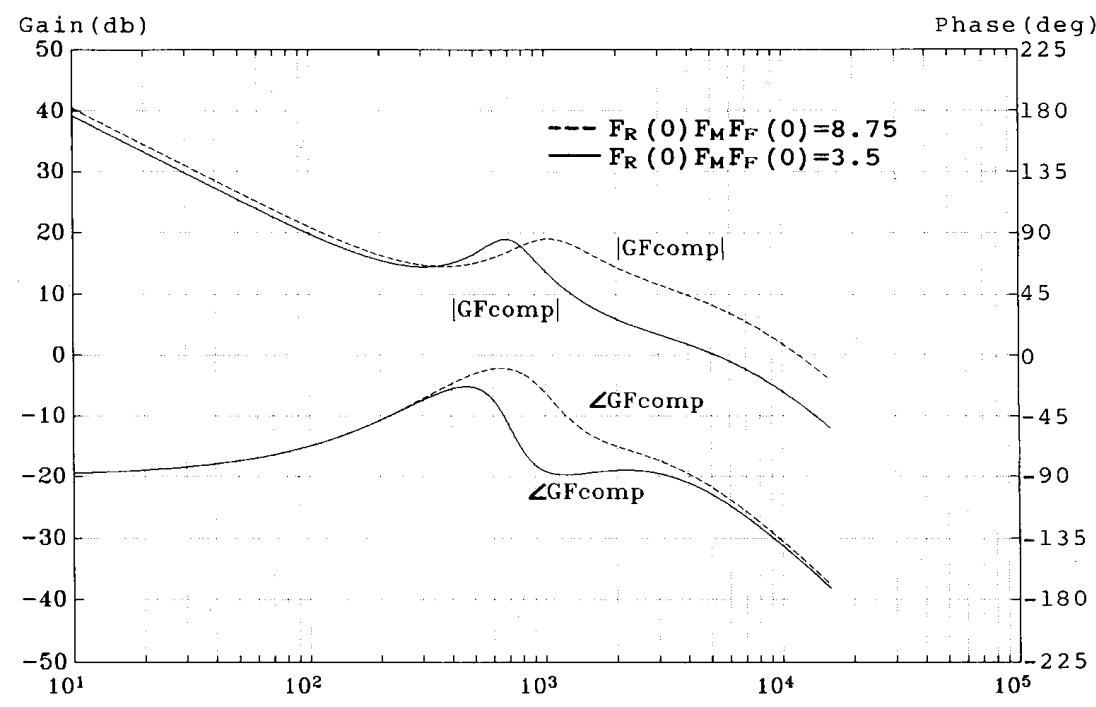

(a)

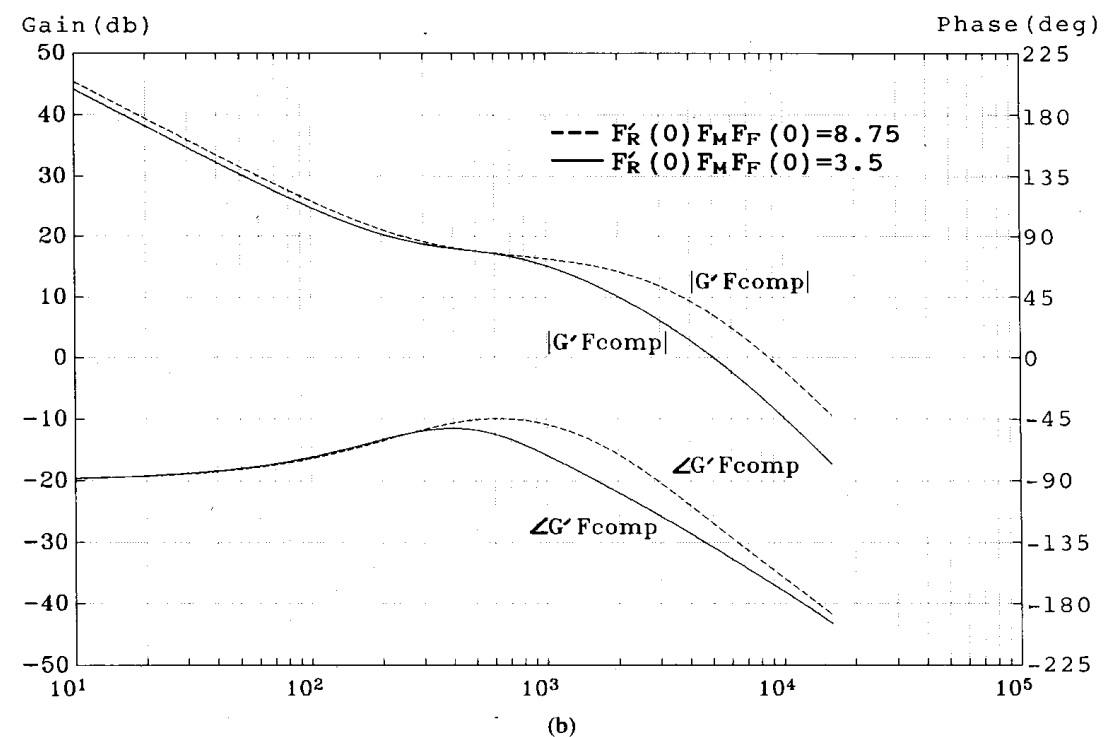

Fig. 9. Predicted loop-gain changes due to saturable reactor variation $\left(R_{C}=0.1 \Omega\right)$. (a) Conventional compensation. (b) Inner loop compensation with lead-lag outer-loop compensation.

source and $\alpha=1$ for a perfect voltage resetting source. In this example, $D^{\prime}=1-V_{o} / V_{x}=0.83$ and $\alpha$ is selected to be 0.2 because this number best fits the experimental results. Fig. 8(b) shows the resultant comparison of predicted values and measured values when the phase delay is taken into consideration. In Figs. 9 to 11 , phase delay is also incorporated in the model. For frequency less than one tenth of the switching frequency, however, the phase delay can be ignored without loss of accuracy.

Comparison of Conventional Compensation Scheme and Inner-Loop Compensation Scheme: Fig. 9 shows the resultant loop gain transfer function using both the conventional compensation scheme and the inner-loop compensation scheme. The circuit parameters used for the solid curves are identical to those used in the Design Example. When the low frequency gain $F_{R^{\prime}}(0) F_{M} F_{F}(0)$ is increased to 8.75 , the loop gain variation is indicated by the dotted curve. As can be seen from this figure, the inner-loop compensation scheme gives smaller loop-gain variation as $F_{R^{\prime}}(0) F_{M} F_{F}(0)$ changes. Among the parameters that may cause $F_{R^{\prime}}(0) F_{M} F_{F}(0)$ change, the reactor average permeability $\mu_{M}$ is most susceptible to unit-to-unit 

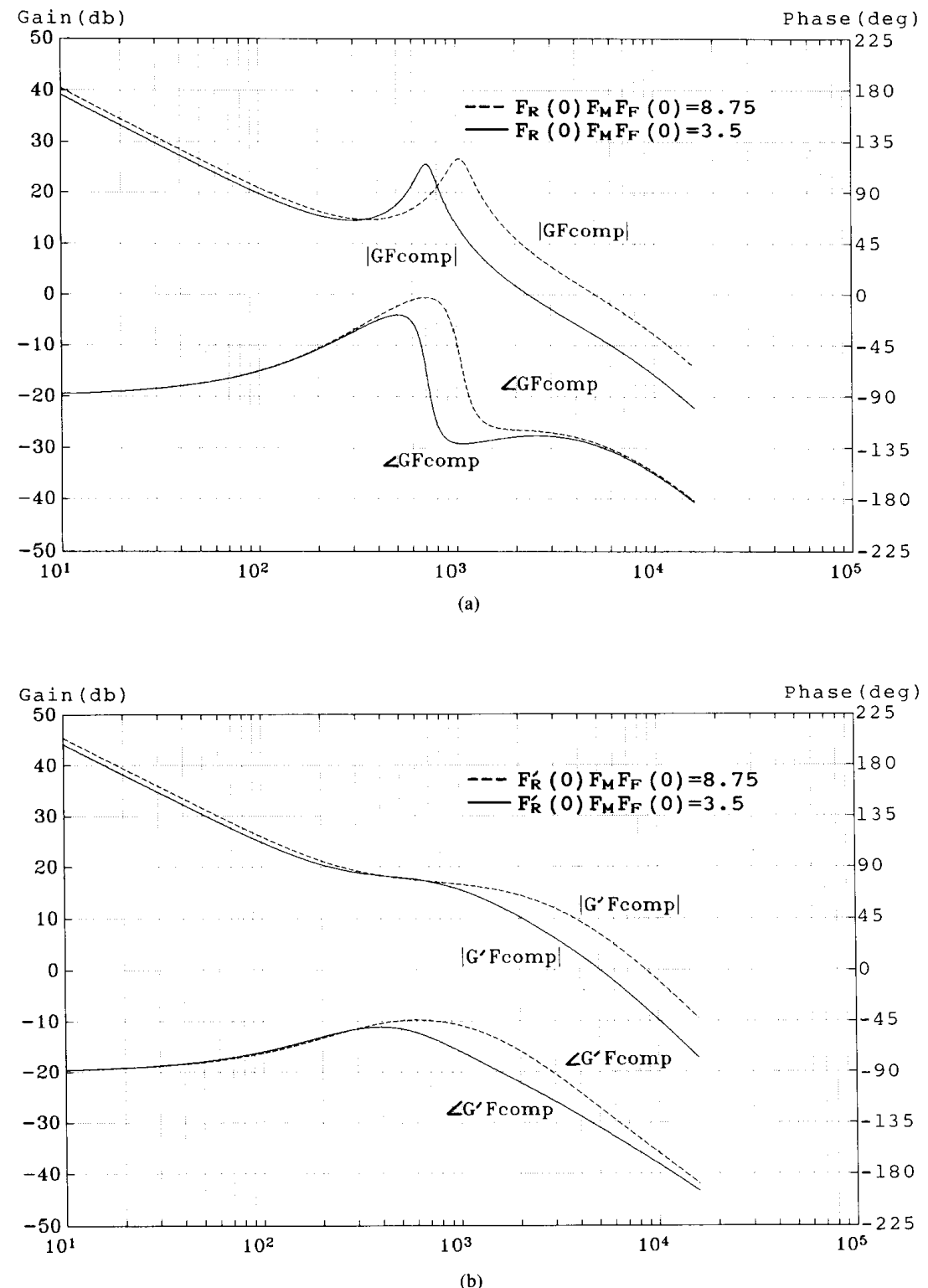

Fig. 10. Predicted loop-gain changes due to saturable reactors variation $\left(R_{C}=0.03 \Omega\right)$. (a) Conventional compensation. (b) Inner-loop compensation with lead-lag outer-loop compensation.

variation. In this case, the performance difference between the two compensation schemes is still not very striking. As capacitor ESR varies, the effect becomes more pronounced as shown below.

Effect of Capacitor ESR: Fig. 10 shows similar comparison as Fig. 9 but the filter capacitor ESR value is reduced from $0.1 \Omega$ to $0.03 \Omega$. The advantages of using the inner-loop compensation are even more pronounced. This is because in the conventional scheme, the capacitor ESR zero in the loop gain function is farther away from the two resonance poles, which makes the resonance effect more pronounced. The phase angle approaches $-180^{\circ}$ before the ESR zero tends to pull it back to- $90^{\circ}$. In the inner-loop compensation scheme, however, there is a compensation zero to dilute the effect of the resonance poles regardless of the ESR zero and consequently ESR zero has relatively little effect on the resultant loop gain performance.

Dominant-Pole-Compensation: Using the same circuit parameters and same crossover frequency requirements as 


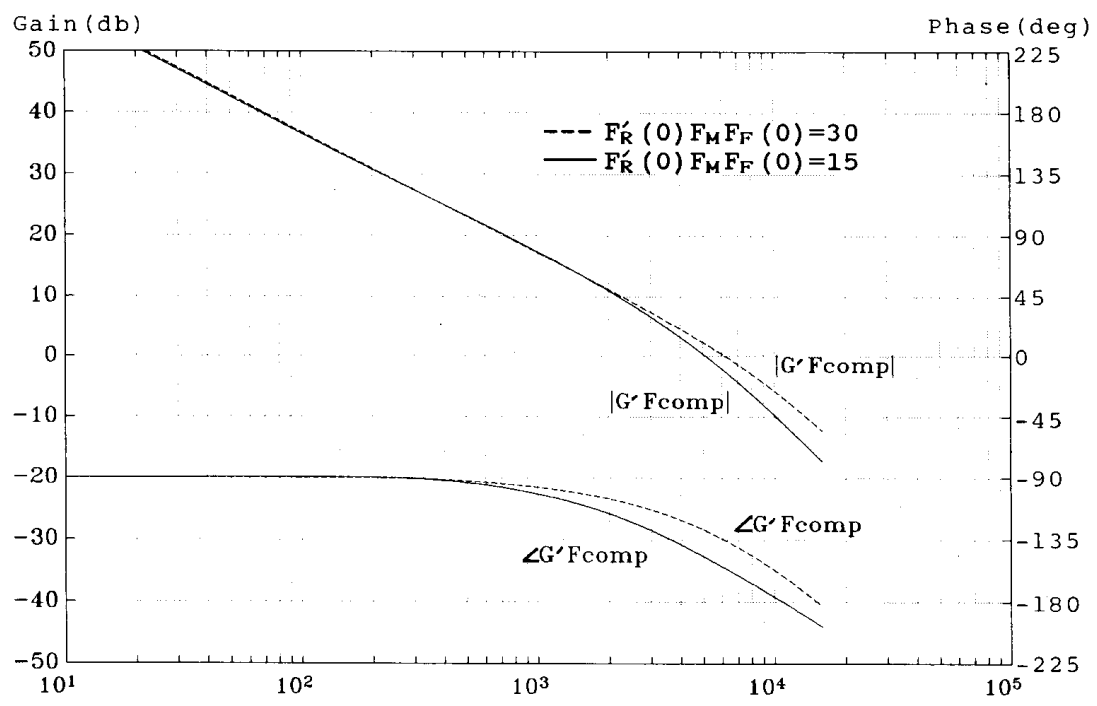

Fig. 11. Loop-gain changes due to saturable reactor variation using dominant pole compensation $\left(f_{C}<f_{P G}\right)$.

the Design Example, one should not use dominant pole compensation. This is because $f_{P G^{\prime}}(=1434 \mathrm{~Hz})$ is much less than $f_{C}(=5 \mathrm{kHz})$. If the inner-loop gain is increased to make $F_{R^{\prime}}(0) F_{M} F_{F}(0)=15$, then $f_{P G} \approx 5 \mathrm{kHz}$ according to (6). Under this condition, a dominant-pole compensation can be used without risking system instability. Fig. 10 shows the resultant loop gain transfer functions. As can be seen from the figure, frequency less than $f_{C}$, the loop gain is essentially independent of parameter variation of the saturable reactor. In other words, the loopgain crossover frequency is essentially independent of power circuit parameter variation.

\section{CONCLUSION}

Using the inner-loop compensation scheme described in this paper, the order of the open-loop transfer function of a magamp control circuit is essentially reduced from two to one for a self-reset scheme. The problem normally associated with compensating a control transfer function with resonance peaking is therefore eliminated. Moreover, if the inner loop gain is designed high enough, the closed-loop gain can be insensitive to the variation of power circuit parameters such as saturable ractor permeability and capacitor ESR, which is a very desirable feature in a high-volume production situation.

A design example is given to illustrate the step-by-step design procedure for a practical magamp control circuit. The results are verified by loop gain measurements.

\section{Nomenclature}

$A_{e} \quad$ Effective cross-section area of the saturable reactor $\left(\mathrm{cm}^{2}\right)$.

$\Delta B$

Flux density swing of the saturable reactor (G).

\begin{tabular}{|c|c|}
\hline$C$ & Capacitance of output filter capacitor (F). \\
\hline$C_{E}$ & Inner loop compensation capacitance $(\mathrm{F})$. \\
\hline$C_{f}, C_{h f}, C_{d}$ & Outer loop compensation capacitance $(\mathrm{F})$. \\
\hline & $\begin{array}{l}\text { Small signal variation of the duty cycle of } \\
\text { saturable reactor (dimensionless). }\end{array}$ \\
\hline$D_{Q}$ & $\begin{array}{l}\text { Main transistor duty cycle (dimension- } \\
\text { less). }\end{array}$ \\
\hline & Frequency $\left(\mathrm{s}^{-1}\right)$ \\
\hline$F_{\text {comp }}$ & $\begin{array}{l}\text { Outer loop compensation transfer function } \\
\text { (dimensionless). }\end{array}$ \\
\hline$f_{C 1}$ & $0 \mathrm{db}$ crossover frequency of $F_{R^{\prime}} F_{M} F_{F}\left(\mathrm{~s}^{-}\right)$. \\
\hline$F_{F}$ & Duty cycle-to-output transfer function (V). \\
\hline$F_{M}$ & $\begin{array}{l}\text { Reset current-to-duty cycle transfer func- } \\
\text { tion or modulator gain of the saturable } \\
\text { reactor }\left(\mathrm{A}^{-1}\right) \text {. }\end{array}$ \\
\hline$f_{P}$ & Pole frequency of $G\left(\mathrm{~s}^{-1}\right)$ \\
\hline$P$ & Pole frequency of $G^{\prime}\left(\mathrm{s}^{-1}\right)$. \\
\hline$f_{P I}$ & $\begin{array}{l}\text { Inner loop compensation pole frequency } \\
\left(\mathrm{s}^{-1}\right) \text {. }\end{array}$ \\
\hline$f_{P O}$ & $\begin{array}{l}\text { Outer loop compensation pole frequency } \\
\left(\mathrm{s}^{-1}\right) \text {. }\end{array}$ \\
\hline$f_{Z I}$ & $\begin{array}{l}\text { Inner loop compensation zero frequency } \\
\left(\mathrm{s}^{-1}\right) \text {. }\end{array}$ \\
\hline$f_{\text {Zo }}$ & $\begin{array}{l}\text { Outer loop compensation zero frequency } \\
\left(\mathrm{s}^{-1}\right) \text {. }\end{array}$ \\
\hline$f_{R}$ & Resonant frequency of $F_{F}$. \\
\hline$F_{R}$ & $\begin{array}{l}\text { Control voltage-to-reset current transfer } \\
\text { function (mho). }\end{array}$ \\
\hline$F_{R^{\prime}}$ & $\begin{array}{l}\text { Control voltage-to-reset current transfer } \\
\text { with inner loop compensated (mho). }\end{array}$ \\
\hline$f_{R G}$ & $\begin{array}{l}\text { Resonant frequency of transfer function } \\
F_{R^{\prime}} F_{M} F_{F} /\left(1+F_{R^{\prime}} F_{M} F_{F}\right)\left(\mathrm{s}^{-1}\right)\end{array}$ \\
\hline $\begin{array}{l}G \\
G^{\prime}\end{array}$ & $\begin{array}{l}F_{R} F_{M} F_{F} /\left(1+F_{R} F_{M} F_{F}\right) \text { (dimensionless). } \\
F_{R^{\prime}} F_{M} F_{F} /\left(1+F_{R^{\prime}} F_{M} F_{F}\right) \text { (dimension- } \\
\quad \text { less). }\end{array}$ \\
\hline
\end{tabular}

$C \quad$ Capacitance of output filter capacitor (F). Inner loop compensation capacitance $(\mathrm{F})$. all signal variation of the duty cycle of less) uter loop compensation transfer function ensionless). Duty cycle-to-output transfer function (V). tion or modulator gain of the saturable actor $\left(\mathrm{A}^{-1}\right)$

Pole frequency of $G\left(\mathrm{~s}^{-1}\right)$

$f_{P G^{\prime}} \quad$ Pole frequency of $G^{\prime}\left(\mathrm{s}^{-1}\right)$ $\left(\mathrm{s}^{-1}\right)$. $\left(\mathrm{s}^{-1}\right)$. $\left(\mathrm{s}^{-1}\right)$. $\left(s^{-1}\right)$.

Resonant frequency of $F_{F}$.

function (mho)

with inner loop compensated (mho).

$F_{R}, F_{M} F_{F} /\left(1+F_{R}, F_{M} F_{F}\right)\left(\mathrm{s}^{-1}\right)$.

作 less). 


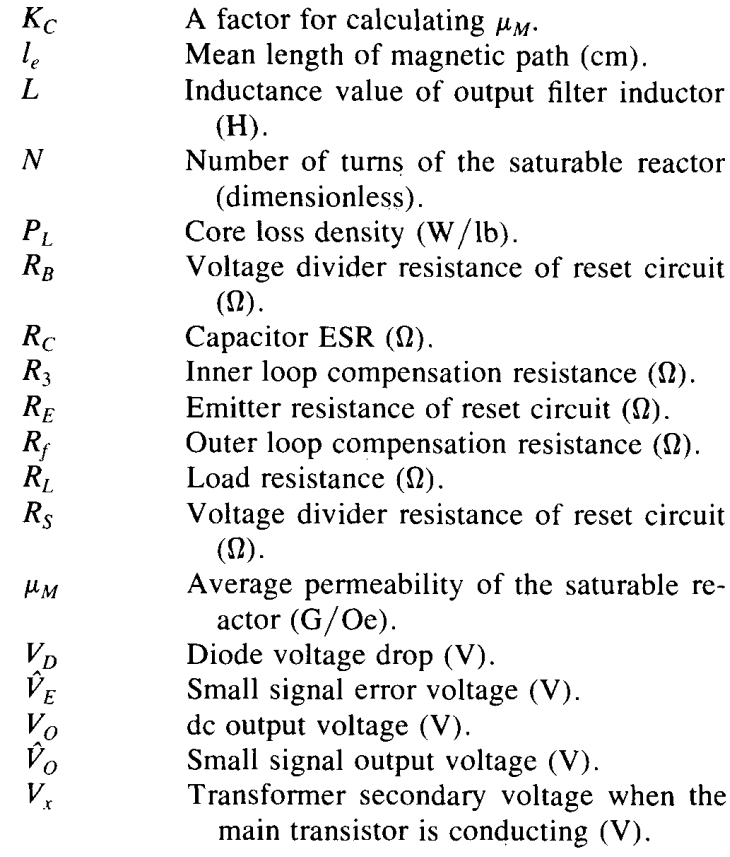

\section{APPENDIX}

\section{A. Behavior of the $G^{\prime}$ Function}

In this section, the behavior of the $G^{\prime}$ function is discussed using phasor diagrams. Fig. 12(a) shows the general shapes of several transfer functions involved from which $G^{\prime}$ function will be derived. Fig. 12(b) shows the resultant $G^{\prime}$ function. There are two assumptions made in this discussion: 1) $F_{R^{\prime}}(0) F_{M} F_{F}(0) \gg 1$, and 2) $f_{C l} \gg$ $f_{R}$. Since $G^{\prime}=F_{R^{\prime}} F_{M} F_{F} /\left(1+F_{R}, F_{M} F_{F}\right)$ and $F_{R} \cdot F_{M} F_{F}$ is a phasor, the behavior of $G^{\prime}$ is investigated by using phase diagrams as follows:

$f<f_{R}$ :

Since $F_{R^{\prime}} F_{M} F_{F} \gg 1$ and $\angle F_{R^{\prime}} F_{M} F_{F} \approx 0^{\circ},\left|G^{\prime}\right|$ is slightly less than 1 , and $\angle G^{\prime}$ is very small.

$f$ is slightly less than $f_{R}$ :

From Fig. 12(a), $0^{\circ}<\left(\angle F_{R^{\prime}}, F_{M} F_{F}\right)<45^{\circ}$. Therefore from Fig. 13(a), $0^{\circ}<\left(\angle G^{\prime}\right)<45^{\circ}$, and $\left|G^{\prime}\right|$ is slightly less than 1 . Normally, $\angle G$ is much smaller than $45^{\circ}$;

$f$ is slightly larger than $f_{R}$ :

From Fig. $12(\mathrm{a}), 0^{\circ}>\left(F_{R}, F_{M} F_{F}\right)>-135^{\circ}$. Therefore, from Fig. 13(b), $\left|G^{\prime}\right|$ can be greater or less than 1 depending upon $\angle F_{R} F_{M} F_{F}$ value. But in either case, $\left|G^{\prime}\right|$ is very close to 1 , and $0^{\circ}>\left(\angle G^{\prime}\right)>-45^{\circ}$. Normally, $\left|\angle G^{\prime}\right|$ is much smaller than $45^{\circ}$;

$f=f_{C l}$ :

From Fig. 12(a), $-90^{\circ}>\left(\angle F_{R} \cdot F_{M} F_{F}\right)>-135^{\circ}$, depending on the relative magnitude of $f_{C 1}$ and $f_{R}$. Therefore, from Fig. 13(c) $-45^{\circ}>\left(\angle G^{\prime}\right)>-67.5^{\circ}$ and $(1 / \sqrt{2})<\left|G^{\prime}\right|<$ a number slightly greater than 1 . Because $f_{C l} \gg f_{R}, \angle G^{\prime}$ is normally closer to $-45^{\circ}$; $f>f_{C 1}$ :

For this frequency range, $F_{R^{\prime}} F_{M} F_{F} \ll 1$ and
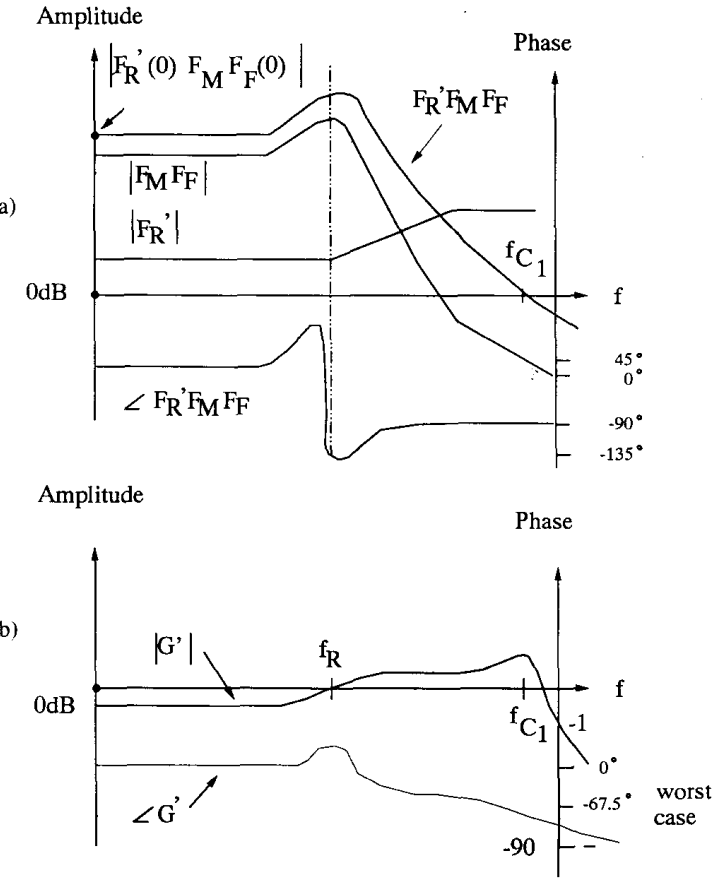

Fig. 12. (a) Bode plots of $F_{R} \cdot F_{M} F_{F}$. (b) Bode plot of $G^{\prime}$.

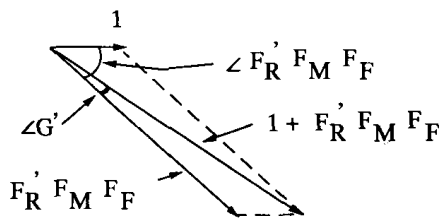

(a)

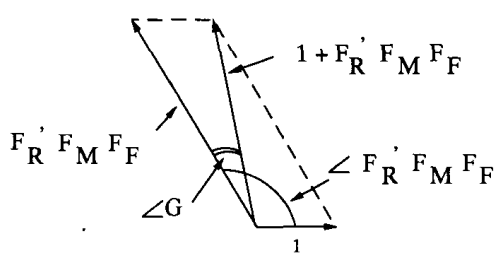

(b)

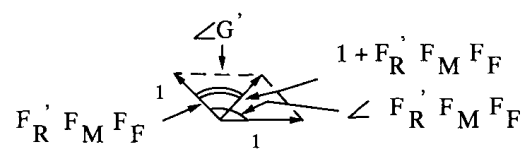

(c)

Fig. 13. Phasor diagrams for studying behavior of $G^{\prime}$ function (a) $f$ is slightly less than $f_{R}$. (b) $f$ is slightly greater than $f_{R}$. (c) $f$ is equal to $f_{C 1}$.

$<F_{R^{\prime}} F_{M} F_{F} \approx 90^{\circ}$. Therefore, $\left|G^{\prime}\right| \cong F_{R^{\prime}}, F_{M} F_{F}$ and $\angle G^{\prime}$ $\approx-90^{\circ}$.

From the above discussion, it can be seen that $G^{\prime}(s)$ can be approximated by a single-pole transfer function 
with pole locating at $f_{C 1}$, i.e., $f_{P G^{\prime}} \approx f_{C 1}$. The plot of $G^{\prime}$ is shown in Fig. 12(b).

\section{B. Derivation of (6)}

The inner-loop transfer function after (inner-loop) compensation can be expressed as follows:

$$
F_{R} F_{M} F_{F}=\frac{K\left(\frac{s}{\omega_{o}}+1\right)}{\frac{s^{2}}{\omega_{o}^{2}}+\frac{1}{Q} \frac{s}{\omega_{o}}+1}
$$

where $K$ is equal to $F_{R^{\prime}}(0) F_{M} F_{F}(0)$, the low frequency gain. $K$ is assumed to be much greater than $1 . Q$ is the quality factor and is assumed to be greater than 1 , and $\omega_{o}$ $=1 / \sqrt{L C}$. From the deriation in Part $A, f_{P G^{\prime}} \cong f_{C 1}$. To find out $f_{\mathrm{Cl}}$, one can proceed as follows:

At $f=f_{C 1}, F_{R^{\prime}} F_{M} F_{F}=0 \mathrm{~dB}=1$. Therefore, set

$$
F_{R}, F_{M} F_{F}=\frac{K\left(\frac{s}{\omega_{o}}+1\right)}{\frac{s^{2}}{\omega_{o}^{2}}+\frac{1}{Q} \frac{s}{\omega_{o}}+1}=1
$$

and solve for the roots $s_{1}$, and $s_{2}$.

$$
s_{1}, s_{2}=\frac{\left(\frac{1}{Q \omega_{o}}-\frac{K}{\omega_{o}}\right) \pm \sqrt{\left(\frac{1}{Q \omega_{o}}-\frac{K}{\omega_{o}}\right)-\frac{4(1-K)}{\omega_{o}}}}{\frac{2}{\omega_{o}^{2}}} .
$$

Since $Q>1$ and $K \gg 1$ and the root must be positive,

$$
s_{1} \cong \frac{\omega_{o} K}{2}[1+\sqrt{1+4 / K}]
$$

Therefore,

$$
\begin{aligned}
f_{P G^{\prime}}= & f_{C 1}=\frac{s_{1}}{2 \pi} \\
= & \frac{1}{2 \pi \sqrt{L C}} \cdot \frac{F_{R^{\prime}}(0) F_{M} F_{F}(0)}{2} \\
& \cdot\left[1+\sqrt{1+\frac{4}{F_{R^{\prime}}(0) F_{M} F_{F}(0)}}\right] .
\end{aligned}
$$

\section{REFERENCES}

[1] I. J. Lee, D. Chen, Y. P. Wu, and C. Jamerson, "Modeling control loop behavior of magamp post regulators," in IEEE Trans. Power Electron, Oct. 1990.

[2] D. Chen, J. Lee, and C. Jamerson, "A simple model predicts small signal control loop behavior of magamp post regulator," IEEE Trans. Power Electron., Oct. 1988.

[3] C. Jamerson, "Calculation of magnetic amplifier post regulator voltage control loop parameters," in High Frequency Power Conversion Conf. Rec., Apr. 1987.

[4] J. Lee, D. Chen, and C. Jamerson, "Magamp post regulators-Practical considerations to allow operation under extreme loading conditions," in IEEE APEC Conf. Rec., Feb. 1988.

[5] R. M. Tedder, "Limitations of the magamp regulator and an improved magamp choke design procedure," in IEEE APEC Conf. Rec., Feb. 1988.

[6] T. Koyashiki and T. Ogata, "Design considerations in multi output dc converter with magnetic amplifiers," in Fifth International Telecommunications Energy Conf. Rec., 83CH1855-6, paper 12-3, Oct. 1983, pp. 388-394.

[7] K. Harada, T. Nabeshima, R. Himastu, and I. Morigoe, "A dc converter controlled by magnetic amplifiers with $1 \mathrm{MHz}$ switching," in IEEE Power Electroncs Specialists Conf. Rec., 5CH2000-8, June 1984, pp. 382-387.

[8] A. Urling, T. Wilson, H. Own, G. Cromwell, and J. Paulakonics, "Modeling the frequency domain behavior of magnetic amplifier controlled high frequency switched mode power supplies," in APEC Rec., 1987.

[9] Using an Integrated Controller in the Design of Magamp Output Regulators, Unitrode Applications Note U-109.

[10] R. Hiramatsu and C. Mullet, "Using saturable reactor control in 500 $\mathrm{kHz}$ converter design,"' Proceedings of the Tenth International Solid State Power Conversion Conf. Rec., paper F2, pp. 1-10, Mar. 1983.

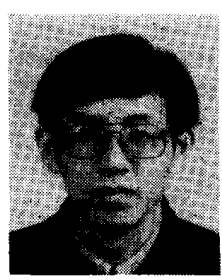

Chao-Hwa Yang received the B.S. degree in electronic engineering from National Taiwan Institute of Technology, the M.S. degree in biomedical engineering from Chung Yuan University, and the Ph.D. degree in electrical engineering from National Taiwan University, Taipei, Taiwan.

$+2$

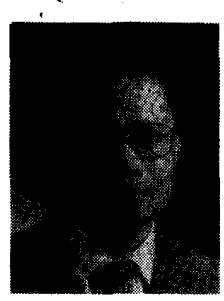

Dan Y. Chen (S'72-M'75-M'79-SM'83) received the B.S. degree from National Chiao-Tung University, Taiwan and the Ph.D. degree from Duke University, Durham, NC, both in electrical engineering, in 1969 and 1975, respectively.

From 1975 to 1979 he was employed as a mem ber of the research staff at the General Electrical Research and Development Center, Schenectady, NY. Since 1979 he has been on the faculty of the Department of Electrical Engineering, Virginia Polytechnic Institute and State University Blacksburg, VA, where he is presently a Professor. His research activities include work in power semiconductor circuits, circuit-device interactions, device characterization, magnetic devices for power electronic applications, and product applications such as brushless motor robotic drive, electronic ballast, appliance power supply, electric car drive, etc. He has published one book and more than 50 papers and has been awarded six U.S. patents in the field of power electronics.

Dr. Chen served as Chairman of the Power Semiconductor Committee of the IEEE Industry Applications Society from 1984-1986.

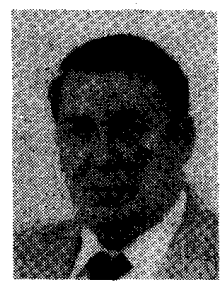

Cliff Jamerson received the B.S.E.E. degree from Purdue University, West Lafayette, IN, and the M.S.E.E. degree from the U.S. Naval PostGraduate School.

He has over ten years experience in power supply design. Prior to entering Purdue he had 20 years in the U.S. Navy. While in the Navy, he coauthored three textbooks and two laboratory manuals. He is presently a Design Engineer with NCR Corporation Power System Division at Lake May. FL.

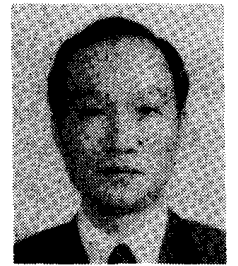

Yan Pei Wu was born in Tainan, Taiwan, the Republic of China, in 1932. He received the B.S degree in electrical engineering from National Taiwan University, Taipei, Tăiwan, the M.S. degree in electronic engineering from National Chiao-Tung University, Shin-Chu, Taiwan, and the $\mathrm{Ph} . \mathrm{D}$. degree in electrical engineering from the University of Washington, Seattle, in 1955 1960 , and 1973 , respectively.

From 1957 to 1961 , he was an Engineer with the Telecommunication Organization, Taiwan, Republic of China. In 1962, he joined the faculty of the Department of Electrical Engineering, National Taiwan University, where he is now a Professor.

Dr. Wu is a member of the Institute of Electrical Engineers of China. 